\title{
Exact energy of the spin-polarized two-dimensional electron gas at high density
}

\author{
Pierre-François Loos* ${ }^{*}$ and Peter M. W. Gill ${ }^{\dagger}$ \\ Research School of Chemistry, Australian National University, Canberra, ACT 0200, Australia \\ (Received 11 March 2011; published 8 June 2011)
}

\begin{abstract}
We derive the exact expansion, to $O\left(r_{s}\right)$, of the energy of the high-density spin-polarized two-dimensional uniform electron gas, where $r_{s}$ is the Seitz radius.
\end{abstract}

DOI: 10.1103/PhysRevB.83.233102

PACS number(s): 71.10.Ca, 73.20.-r, 31.15.E-

The three-dimensional uniform electron gas is a ubiquitous paradigm in solid-state physics ${ }^{1}$ and quantum chemistry, ${ }^{2}$ and has been extensively used as a starting point in the development of exchange-correlation density functionals in the framework of density-functional theory. ${ }^{3}$ The two-dimensional version of the electron gas has also been the object of extensive research ${ }^{4,5}$ because of its intimate connection to two-dimensional or quasi-two-dimensional materials, such as quantum dots. ${ }^{6,7}$

The two-dimensional gas (or 2-jellium) is characterized by a density $\rho=\rho_{\uparrow}+\rho_{\downarrow}$, where $\rho_{\uparrow}$ and $\rho_{\downarrow}$ are the (uniform) densities of the spin-up and spin-down electrons, respectively. In order to guarantee its stability, the electrons are assumed to be embedded in a uniform background of positive charge. ${ }^{8}$ We will use atomic units throughout.

It is known from contributions by numerous workers ${ }^{9-19}$ that the high-density (i.e., small $r_{s}$ ) expansion of the energy per electron (or reduced energy) in 2-jellium is

$$
\begin{aligned}
E\left(r_{s}, \zeta\right)= & \frac{\varepsilon_{-2}(\zeta)}{r_{s}^{2}}+\frac{\varepsilon_{-1}(\zeta)}{r_{s}}+\varepsilon_{0}(\zeta) \\
& +\varepsilon_{\ell}(\zeta) r_{s} \ln r_{s}+O\left(r_{s}\right)
\end{aligned}
$$

where $r_{s}=(\pi \rho)^{-1 / 2}$ is the Seitz radius, and

$$
\zeta=\frac{\rho_{\uparrow}-\rho_{\downarrow}}{\rho}
$$

is the relative spin polarization. ${ }^{8}$ Without loss of generality, we assume $\rho_{\downarrow} \leqslant \rho_{\uparrow}$, i.e., $\zeta \in[0,1]$.

The first two terms of the expansion (1) are the kinetic and exchange energies, and their sum gives the Hartree-Fock (HF) energy. The paramagnetic $(\zeta=0)$ coefficients are

$$
\begin{gathered}
\varepsilon_{-2}(0)=+\frac{1}{2}, \\
\varepsilon_{-1}(0)=-\frac{4 \sqrt{2}}{3 \pi},
\end{gathered}
$$

and their spin-scaling functions are

$$
\begin{gathered}
\Upsilon_{-2}(\zeta)=\frac{\varepsilon_{-2}(\zeta)}{\varepsilon_{-2}(0)}=\frac{(1-\zeta)^{2}+(1+\zeta)^{2}}{2} \\
\Upsilon_{-1}(\zeta)=\frac{\varepsilon_{-1}(\zeta)}{\varepsilon_{-1}(0)}=\frac{(1-\zeta)^{3 / 2}+(1+\zeta)^{3 / 2}}{2}
\end{gathered}
$$

In this Brief Report, we show that the next two terms, which dominate the expansion of the reduced correlation energy, ${ }^{20}$ can also be obtained in closed form for any value of the relative spin polarization $\zeta$.
The logarithmic coefficient $\varepsilon_{\ell}(\zeta)$ can be obtained by a GellMann-Brueckner resummation ${ }^{21}$ of the most divergent terms in the infinite series in Eq. (1), and this yields ${ }^{13}$

$$
\varepsilon_{\ell}(\zeta)=-\frac{1}{12 \sqrt{2} \pi} \int_{-\infty}^{\infty}\left[R\left(\frac{u}{k_{\uparrow}}\right)+R\left(\frac{u}{k_{\downarrow}}\right)\right]^{3} d u,
$$

where

$$
R(u)=1-\frac{1}{\sqrt{1+1 / u^{2}}}
$$

and

$$
k_{\uparrow, \downarrow}=\sqrt{1 \pm \zeta}
$$

is the Fermi wave vector associated with the spin-up and spindown electrons, respectively. After an unsuccessful attempt by $\mathrm{Zia},{ }^{11}$ the paramagnetic $(\zeta=0)$ and ferromagnetic $(\zeta=1)$ values,

$$
\begin{gathered}
\varepsilon_{\ell}(0)=-\sqrt{2}\left(\frac{10}{3 \pi}-1\right)=-0.0863136 \ldots, \quad \\
\varepsilon_{\ell}(1)=\frac{1}{4 \sqrt{2}} \varepsilon_{\ell}(0)=-\frac{1}{4}\left(\frac{10}{3 \pi}-1\right)=-0.0152582 \ldots,
\end{gathered}
$$

were found by Rajagopal and Kimball ${ }^{13}$ and the spin-scaling function,

$$
\Upsilon_{\ell}(\zeta)=\frac{\varepsilon_{\ell}(\zeta)}{\varepsilon_{\ell}(0)}=\frac{1}{8}\left[k_{\uparrow}+k_{\downarrow}+3 \frac{F\left(k_{\uparrow}, k_{\downarrow}\right)+F\left(k_{\downarrow}, k_{\uparrow}\right)}{10-3 \pi}\right],
$$

was obtained 30 years later by Chesi and Giuliani. ${ }^{18}$ The explicit expression for $F(x, y)$ is

$$
F(x, y)=4(x+y)-\pi x-4 x E\left(1-\frac{y^{2}}{x^{2}}\right)+2 x^{2} \kappa(x, y),
$$

where

$$
\kappa(x, y)= \begin{cases}\left(x^{2}-y^{2}\right)^{-1 / 2} \arccos (y / x), & x \leqslant y, \\ \left(y^{2}-x^{2}\right)^{-1 / 2} \operatorname{arccosh}(x / y), & x>y,\end{cases}
$$

and $E(x)$ is the complete elliptic integral of the second kind. ${ }^{22}$

The constant coefficient $\varepsilon_{0}(\zeta)$ can be written as the sum

$$
\varepsilon_{0}(\zeta)=\varepsilon_{0}^{\mathrm{a}}(\zeta)+\varepsilon_{0}^{\mathrm{b}}
$$


of a direct ("ring-diagram") term $\varepsilon_{0}^{\mathrm{a}}(\zeta)$ and an exchange term $\varepsilon_{0}^{\mathrm{b}}$. Following Onsager's work ${ }^{23}$ on the three-dimensional gas, the exchange term was found by Isihara and Ioriatti ${ }^{14}$ to be

$$
\varepsilon_{0}^{\mathrm{b}}=\beta(2)-\frac{8}{\pi^{2}} \beta(4)=+0.114357 \ldots,
$$

where $\beta$ is the Dirichlet beta function ${ }^{22}$ and $G=\beta(2)$ is Catalan's constant. We note that $\varepsilon_{0}^{\mathrm{b}}$ is independent of $\zeta$ and the spin-scaling function therefore takes the trivial form

$$
\Upsilon_{0}^{\mathrm{b}}(\zeta)=\frac{\varepsilon_{0}^{\mathrm{b}}(\zeta)}{\varepsilon_{0}^{\mathrm{b}}(0)}=1
$$

The direct term has not been found in closed form, but we now show how this can be achieved. Following Rajagopal and Kimball, ${ }^{13}$ we write the direct term as the double integral

$$
\begin{aligned}
\varepsilon_{0}^{\mathrm{a}}(\zeta)= & -\frac{1}{8 \pi^{3}} \int_{-\infty}^{\infty} \int_{0}^{\infty}\left[Q_{q / k_{\uparrow}}\left(\frac{u}{k_{\uparrow}}\right)\right. \\
& \left.+Q_{q / k_{\downarrow}}\left(\frac{u}{k_{\downarrow}}\right)\right]^{2} d q d u,
\end{aligned}
$$

where

$$
\begin{aligned}
Q_{q}(u)= & \frac{\pi}{q}\left[q-\sqrt{\left(\frac{q}{2}-i u-1\right)\left(\frac{q}{2}-i u+1\right)}\right. \\
& \left.-\sqrt{\left(\frac{q}{2}+i u-1\right)\left(\frac{q}{2}+i u+1\right)}\right] .
\end{aligned}
$$

In the paramagnetic $(\zeta=0)$ case, the transformation $s=$ $q^{2} / 4-u^{2}$ and $t=q u$ yields

$$
\begin{aligned}
\varepsilon_{0}^{\mathrm{a}}(0)= & -\frac{1}{2 \pi} \int_{-\infty}^{\infty} \int_{0}^{\infty} \frac{1}{\sqrt{s^{2}+t^{2}}} \\
& \times\left[1-\left(\frac{\sqrt{(s-1)^{2}+t^{2}}+s-1}{\sqrt{s^{2}+t^{2}}+s}\right)^{1 / 2}\right]^{2} d t d s
\end{aligned}
$$

and, if we adopt polar coordinates, this becomes

$$
\begin{aligned}
\varepsilon_{0}^{\mathrm{a}}(0) & =-\frac{1}{2 \pi} \int_{0}^{\infty} \int_{0}^{\pi}\left[1-\sqrt{\frac{\sqrt{1-2 r \cos \theta+r^{2}}-1+r \cos \theta}{r(1+\cos \theta)}}\right]^{2} d \theta d r \\
& =-\frac{1}{2 \pi} \int_{0}^{\pi}\left[2 \ln 2-(\pi-\theta) \tan \frac{\theta}{2}-2 \tan ^{2} \frac{\theta}{2} \ln \left(\sin \frac{\theta}{2}\right)\right] d \theta=\ln 2-1=-0.306853 \ldots
\end{aligned}
$$

which confirms Seidl's numerical estimate ${ }^{17}$

$$
\varepsilon_{0}^{\mathrm{a}}(0)=-0.30682 \pm 0.00012 .
$$

In the ferromagnetic $(\zeta=1)$ case, Eq. (18) yields

$$
\varepsilon_{0}^{\mathrm{a}}(1)=\frac{1}{2} \varepsilon_{0}^{\mathrm{a}}(0)=\frac{\ln 2-1}{2}=-0.153426 \ldots
$$

In intermediate cases, where $0<\zeta<1$, we define the spinscaling function

$$
\Upsilon_{0}^{\mathrm{a}}(\zeta)=\frac{\varepsilon_{0}^{\mathrm{a}}(\zeta)}{\varepsilon_{0}^{\mathrm{a}}(0)}
$$

and, from (18), we have

$$
\Upsilon_{0}^{\mathrm{a}}(\zeta)=\frac{1}{2}-\frac{1}{4 \pi(\ln 2-1)} \int_{0}^{\infty} \int_{-1}^{1} P_{k_{\uparrow}}(r, z) P_{k_{\downarrow}}(r, z) \frac{i d z}{z} d r
$$

where

$$
P_{k}(r, z)=1-\frac{\sqrt{r z-k^{2}}+\sqrt{r / z-k^{2}}}{\sqrt{r}(\sqrt{z}+1 / \sqrt{z})} .
$$

Integrating over $r$ gives

$$
\Upsilon_{0}^{\mathrm{a}}(\zeta)=\frac{1}{2}-\frac{1}{4 \pi(\ln 2-1)} \int_{-1}^{1} L_{k_{\uparrow}, k_{\downarrow}}(z) \frac{i d z}{z},
$$

where

$$
\begin{aligned}
L_{k_{\uparrow}, k_{\downarrow}}(z)= & -k_{\uparrow} \ln k_{\uparrow}-k_{\downarrow} \ln k_{\downarrow}+\frac{1}{(z+1)^{2}}\left[\left(z k_{\uparrow}-k_{\downarrow}\right)^{2}\right. \\
& \times \ln \left(z k_{\uparrow}-k_{\downarrow}\right)+\left(z k_{\downarrow}-k_{\uparrow}\right)^{2} \ln \left(z k_{\downarrow}-k_{\uparrow}\right) \\
& -i \pi\left(k_{\downarrow}^{2}-2 z k_{\uparrow} k_{\downarrow}+k_{\downarrow}^{2}\right)+2 z\left(k_{\uparrow}+k_{\downarrow}\right)^{2} \ln \left(k_{\uparrow}\right. \\
& \left.\left.+k_{\downarrow}\right)-z\left(z k_{\uparrow}^{2}-2 k_{\uparrow} k_{\downarrow}+z k_{\downarrow}^{2}\right) \ln z\right],
\end{aligned}
$$

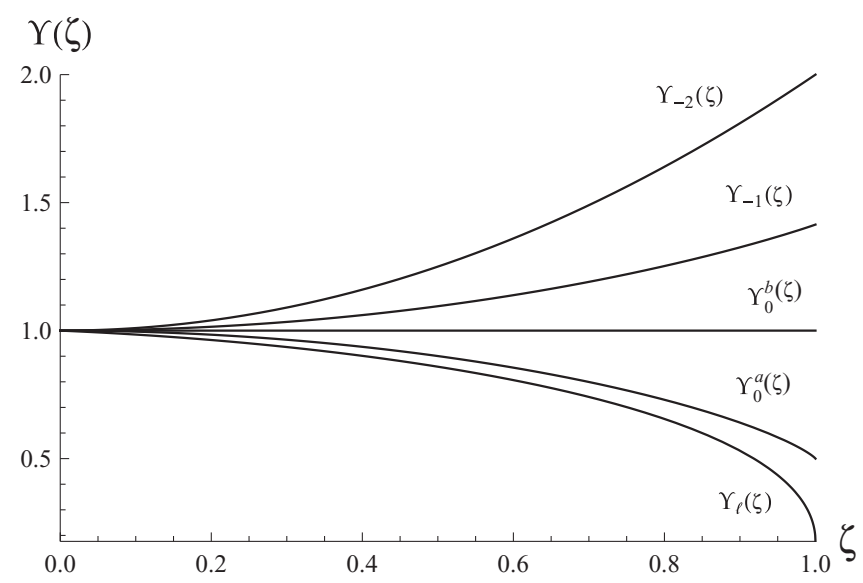

FIG. 1. $\Upsilon_{-2}(\zeta), \Upsilon_{-1}(\zeta), \Upsilon_{0}^{\mathrm{a}}(\zeta), \Upsilon_{0}^{\mathrm{b}}(\zeta)$, and $\Upsilon_{\ell}(\zeta)$ as functions of $\zeta$. 
TABLE I. Energy coefficients and spin-scaling functions for 2-jellium in the high-density limit.

\begin{tabular}{lcccc}
\hline \hline Term & Coefficient & $\varepsilon(0)$ & $\varepsilon(1)$ & $\Upsilon(\zeta)$ \\
\hline$r_{s}^{-2}$ & $\varepsilon_{-2}(\zeta)$ & $\frac{1}{2}$ & 1 & Eq. (5) \\
$r_{s}^{-1}$ & $\varepsilon_{-1}(\zeta)$ & $-\frac{4 \sqrt{2}}{3 \pi}$ & $-\frac{8}{3 \pi}$ & Eq. (6) \\
$r_{s}^{0}$ & $\varepsilon_{0}^{\mathrm{a}}(\zeta)$ & $\ln 2-1$ & $\frac{\ln 2-1}{2}$ & Eq. (29) \\
& $\varepsilon_{0}^{\mathrm{b}}(\zeta)$ & $\beta(2)-\frac{8}{\pi^{2}} \beta(4)$ & $\beta(2)-\frac{8}{\pi^{2}} \beta(4)$ & 1 \\
$r_{s} \ln r_{s}$ & $\varepsilon_{\ell}(\zeta)$ & $-\sqrt{2}\left(\frac{10}{3 \pi}-1\right)$ & $-\frac{1}{4}\left(\frac{10}{3 \pi}-1\right)$ & Eq. (12) \\
\hline \hline
\end{tabular}

and contour integration over $z$ eventually yields

$$
\Upsilon_{0}^{\mathrm{a}}(\zeta)=\frac{1}{2}+\frac{1-\zeta}{4(\ln 2-1)}\left[2 \ln 2-1-\sqrt{\frac{1+\zeta}{1-\zeta}}+\frac{1+\zeta}{1-\zeta}\right.
$$

$$
\left.\times \ln \left(1+\sqrt{\frac{1-\zeta}{1+\zeta}}\right)-\ln \left(1+\sqrt{\frac{1+\zeta}{1-\zeta}}\right)\right] .
$$

This is plotted in Fig. 1 and agrees well with Seidl's approximation, ${ }^{17}$ deviating by a maximum of 0.0005 near $\zeta=0.9815$.

In conclusion, we have shown that the energy of the high-density spin-polarized two-dimensional uniform electron gas can be found in closed form up to $O\left(r_{s}\right)$. We believe that these results, which are summarized in Table I, will be useful in the future development of exchange-correlation functionals within density-functional theory.

We thank Prof. Stephen Taylor for helpful discussions. P.M.W.G. thanks the NCI National Facility for a generous grant of supercomputer time and the Australian Research Council (Grants DP0984806 and DP1094170) for funding. *loos@rsc.anu.edu.au

†Corresponding author: peter.gill@anu.edu.au

${ }^{1}$ W. Kohn, Rev. Mod. Phys. 71, 1253 (1999).

${ }^{2}$ J. A. Pople, Rev. Mod. Phys. 71, 1267 (1999).

${ }^{3}$ R. G. Parr and W. Yang, Density Functional Theory for Atoms and Molecules (Oxford University Press, 1989).

${ }^{4}$ T. Ando, A. B. Fowler, and F. Stern, Rev. Mod. Phys. 54, 437 (1982).

${ }^{5}$ E. Abrahams, S. V. Kravchenko, and M. P. Sarachik, Rev. Mod. Phys. 73, 251 (2001).

${ }^{6}$ Y. Alhassid, Rev. Mod. Phys. 72, 895 (2000).

${ }^{7}$ S. M. Reimann and M. Manninen, Rev. Mod. Phys. 74, 1283 (2002).

${ }^{8}$ G. F. Giuliani and G. Vignale, Quantum Theory of Electron Liquid (Cambridge University Press, Cambridge, 2005).

${ }^{9}$ S. Misawa, Phys. Rev. 140, A1645 (1965).

${ }^{10}$ F. Stern, Phys. Rev. Lett. 30, 278 (1973).

${ }^{11}$ R. K. P. Zia, J. Phys. C 6, 3121 (1973).
${ }^{12}$ A. Isihara and T. Toyoda, Ann. Phys. 106, 394 (1977).

${ }^{13}$ A. K. Rajagopal and J. C. Kimball, Phys. Rev. B 15, 2819 (1977).

${ }^{14}$ A. Isihara and L. Ioriatti, Phys. Rev. B 22, 214 (1980).

${ }^{15}$ B. Tanatar and D. M. Ceperley, Phys. Rev. B 39, 5005 (1989).

${ }^{16} \mathrm{C}$. Attaccalite, S. Moroni, P. Gori-Giorgi, and G. B. Bachelet, Phys. Rev. Lett. 88, 256601 (2002).

${ }^{17}$ M. Seidl, Phys. Rev. B 70, 073101 (2004).

${ }^{18}$ S. Chesi and G. F. Giuliani, Phys. Rev. B 75, 153306 (2007).

${ }^{19}$ N. D. Drummond and R. J. Needs, Phys. Rev. Lett. 102, 126402 (2009).

${ }^{20}$ E. Wigner, Phys. Rev. 46, 1002 (1934).

${ }^{21}$ M. Gell-Mann and K. A. Brueckner, Phys. Rev. 106, 364 (1957).

${ }^{22}$ NIST Handbook of Mathematical Functions, edited by F. W. J. Olver, D. W. Lozier, R. F. Boisvert, and C. W. Clark (Cambridge University Press, New York, 2010).

${ }^{23}$ L. Onsager, L. Mittag, and M. J. Stephen, Ann. Phys. 18, 71 (1966). 